\title{
Design and Power Flow Analysis of Electrical System Using Electrical Transient and Program Software
}

\author{
Raheel Muzzammel*, Ibrahim Khail, Muhammad Huzaifa Tariq, \\ Abubakar Muhammad Asghar, Ali Hassan \\ Department of Electrical Engineering, University of Lahore, Lahore, Pakistan \\ Email: *raheelmuzzammel@gmail.com
}

How to cite this paper: Muzzammel, R., Khail, I., Tariq, M.H., Asghar, A.M. and Hassan, A. (2019) Design and Power Flow Analysis of Electrical System Using Electrical Transient and Program Software. Energy and Power Engineering, 11, 186-199. https://doi.org/10.4236/epe.2019.114011

Received: March 17, 2019

Accepted: April 21, 2019

Published: April 24, 2019

Copyright ( 2019 by author(s) and Scientific Research Publishing Inc. This work is licensed under the Creative Commons Attribution International License (CC BY 4.0).

http://creativecommons.org/licenses/by/4.0/ (c) (i) Open Access

\begin{abstract}
Power flow analysis is a numerical way of study of behavior of flow of electric power in an interconnected system. In order to meet the growing demands of electrical energy in an optimum way, there is a need to upgrade existing systems or to install new systems. Therefore, planning of new installations and determination of best operating conditions of existing systems need power flow analysis. In this way, cost/benefit ratio for both suppliers and customers is maintained. This research involves the design and power flow analysis of IEEE-14 bus system. Newton Raphson method is applied for better efficiency and reduced computational time. Simulation analysis is conducted in ETAP software because of its excessive used in real life systems.
\end{abstract}

\section{Keywords}

Power Flow Analysis, IEEE-14 Bus System, Newton Raphson Method, ETAP Software

\section{Introduction}

Behavior of flow of electric power in an interconnected system is studied numerically by power flow analysis. Single line diagram and per unit system are the fundamental components of this analysis. Voltage $(V)$, voltage angles $(\delta)$, active power $(P)$ and reactive power $(Q)$ are the variables involved in this study. Active power and voltage are known at supply side and reactive power and voltage angles are determined through numerical analysis of power flow. Active power and reactive power are known at consumer side and voltage and voltage angles are evaluated through power flow analysis [1] [2] [3]. 
There is a requirement to improve current power system or to add new systems to existing systems for meeting energy demands. Therefore, power flow analysis is an essential study for future expansion of power system and for finding the ideal operating conditions of existing electric power systems. Moreover, economic dispatch, unit commitment, contingency analysis, transient and steady state analysis and short circuit analysis require power flow analysis [2] [3].

In power flow analysis, there are three types of buses i.e., swing bus, generator bus and load bus. Swing bus and generator bus are source buses. For swing bus, $V$ and $\delta$ are known and $P$ and $Q$ are unknown values. The source bus with larger size of generator is normally taken as swing bus. For generator bus ( $P V$ bus), $P$ and $V$ are known values and $Q$ and $\delta$ are unknown. For load bus ( $P Q$ bus), $P$ and $Q$ are known and $V$ and $\delta$ are unknown values. The unknown parameters of buses (i.e., $P$ and $Q$ for swing bus, $Q$ and $\delta$ for generator bus and $V$ and $\delta$ for load bus) are determined from known values of buses (i.e., $V$ and $\delta$ for swing bus, $P$ and $V$ for generator bus and $P$ and $Q$ for load bus) and impedance between these buses $\left(Z_{i j}\right)$ using power flow calculations. After performing power flow analysis, all the four parameters $(V, \delta, P$ and $Q)$ are available for swing bus, generator bus and load bus. The other electrical parameters such as current, power factor, apparent power for the whole electrical system can be determined from these four parameters $(V, \delta, P$ and $Q$ ) and impedance between the buses $\left(Z_{i j}\right)[2][3]$.

Commercial power systems are complicated. It is not possible to analyze power flow through hand calculations. Physical models of power systems were analyzed through network analyzers in laboratories between 1929 and early 1960. Afterwards, invention of digital computers replaced the analog methods with numerical methods [1]-[7].

Initially, linear methods were proposed to analyze power flow analysis. Among these, Cramer's method, Gauss elimination and $L U$ factorization are notable. However, these methods cannot handle complex, nonlinear and big power systems. Therefore, iterative techniques i.e., Gauss Seidel method, Newton Raphson method are developed to solve complex power systems [4] [5] [6] [7] [8].

In this research paper, Newton Raphson method is implemented because of its accuracy and reduced computational time due to less iteration as compared to Gauss Seidel method. Iterative methods are techniques for solving the $n$ equations of the linear system $A x=b$ one at a time in sequence, and use previously computed results as soon as they are available, In Gauss Seidel method, the computations appear to be serial. Further, each component of new iteration depends upon all previously computed components. Updates cannot be done simultaneously. In addition to this, new iteration depends on the order in which equations are examined. If this ordering is changed, the components of new iteration (and not just their order) also change. These limitations persuade engineers and researchers to go for Newton Raphson method. Gauss Seidel method is easy to program. Newton Raphson method is complex to program and it ac- 
quires more memory space then Gauss Seidel method. Time required for per iteration in Newton Raphson method is larger than Gauss Seidel method but the overall time for iterative process is less because of less number of iterations for convergence [1] [2] [3] [4] [7] [8] [9].

ETAP software is used for simulation because of its extension of real time intelligent power management systems for monitoring, controlling, automating and optimizing power systems. It is a high impact software used for power flow analysis is generation, transmission and distribution systems of electric power engineering [10] [11] [12] [13].

This research paper consists of following sections: Section II covers the mathematical background and proposed flow chart for Newton Raphson method. Section III contains the details of IEEE-14 bus system as a test model for depicting accuracy of power flow analysis method. Simulation analysis in tabular form is presented in Section IV. Section V concludes the research.

\section{Newton Raphson Method}

Newton Raphson method is named after Isaac Newton and Joseph Raphson. Successively better approximations for the roots of a real-valued function are determined from this method. Newton-Raphson load flow analysis is based on Newton Raphson method for evaluation of a system of nonlinear equations. It is an iterative method which approximates a set of non-linear simultaneous equations is approximated to a set of linear simultaneous equations using Taylor's series expansion by this iterative method [2] [3].

Referring to Figure 1, polar form of power flow equations are formulated for $n$ bus system in terms of bus admittance matrix $Y$ as

$$
\begin{gathered}
I_{i}=\sum_{j=1}^{n} Y_{i j} V_{j} \\
I_{i}=\sum_{j=1}^{n}|| Y_{i j}|| V_{j} \mid<\left(\theta_{i j}+\delta_{j}\right)
\end{gathered}
$$

where $i$ and $j$ represent $i^{\text {th }}$ and $j^{\text {th }}$ bus respectively. The current in terms of the active and the reactive power at bus $i$ is:

$$
I_{i}=\frac{P_{i}-J Q_{i}}{V_{i}}
$$

Using Equation (3) into Equation (2) gives:

$$
\begin{gathered}
P_{i}-j Q_{i}=\left|V_{i}\right|<-\delta_{i} \sum_{i=1}^{n}\left|V_{j}\right|\left|Y_{i j}\right|<\theta_{i j}+\delta_{j} \\
P_{i}-j Q_{i}=\sum_{i=1}^{n}\left|V_{i} V_{j} Y_{i j}\right|<\theta_{i j}+\delta_{j}-\delta_{i}
\end{gathered}
$$

Real and imaginary parts are separated as:

$$
\begin{gathered}
P_{i}=\sum_{i=1}^{n}\left|V_{i} V_{j} Y_{i j}\right| \cos \left(\theta_{i j}+\delta_{j}-\delta_{i}\right) \\
Q_{i}=-\sum_{i=1}^{n}\left|V_{i} V_{j} Y_{i j}\right| \sin \left(\theta_{i j}+\delta_{j}-\delta_{i}\right)
\end{gathered}
$$

Taylor series expansion of Equation (6) and Equation (7) and neglecting higher order term result: 


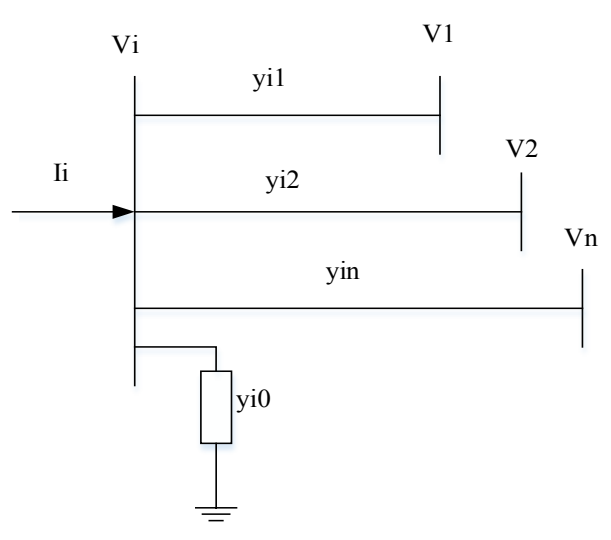

Figure 1. Typical power system bus bar model.

$$
\left[\begin{array}{cccccc}
\frac{\partial P_{2}}{\partial \delta_{2}} & \cdots & \frac{\partial P_{2}}{\partial \delta_{n}} & \frac{\partial P_{2}}{\partial\left|V_{2}\right|} & \cdots & \frac{\partial P_{2}}{\partial\left|V_{n}\right|} \\
\vdots & \ddots & \vdots & \vdots & \ddots & \vdots \\
\frac{\partial P_{n}}{\partial \delta_{2}} & \cdots & \frac{\partial P_{n}}{\partial \delta_{n}} & \frac{\partial P_{2}}{\partial\left|V_{2}\right|} & \cdots & \frac{\partial P_{2}}{\partial\left|V_{2}\right|} \\
\frac{\partial Q_{2}}{\partial \delta_{2}} & \cdots & \frac{\partial Q_{2}}{\partial \delta_{n}} & \frac{\partial Q_{2}}{\partial\left|V_{2}\right|} & \cdots & \frac{\partial Q_{2}}{\partial\left|V_{n}\right|} \\
\vdots & \ddots & \vdots & \vdots & \ddots & \vdots \\
\frac{\partial Q_{n}}{\partial \delta_{2}} & \cdots & \frac{\partial Q_{n}}{\partial \delta_{n}} & \frac{\partial Q_{n}}{\partial\left|V_{2}\right|} & \cdots & \frac{\partial Q_{n}}{\partial\left|V_{n}\right|}
\end{array}\right]\left[\begin{array}{c}
\Delta \delta_{2} \\
\vdots \\
\vdots \\
\Delta \delta_{n} \\
\Delta\left|V_{2}\right| \\
\vdots \\
\vdots \\
\Delta\left|V_{n}\right|
\end{array}\right]=\left[\begin{array}{c}
\Delta P_{2} \\
\vdots \\
\vdots \\
\Delta P_{n} \\
\Delta Q_{n} \\
\vdots \\
\vdots \\
\Delta Q_{n}
\end{array}\right]
$$

Linearized relationship is obtained by this Jacobian matrix between small changes in voltage angles $\Delta \delta_{i}$ and voltage magnitude $\Delta\left|V_{i}\right|$ with the small changes in real and reactive power $\Delta P_{i}$ and $\Delta Q_{i}$. (8) can be modified as:

$$
\left[\begin{array}{c}
\Delta P \\
\Delta Q
\end{array}\right]=\left[\begin{array}{ll}
J_{1} & J_{2} \\
J_{3} & J_{4}
\end{array}\right]\left[\begin{array}{c}
\Delta \delta \\
\Delta|V|
\end{array}\right]
$$

The diagonal and the off diagonal elements of $J_{1}$ are:

$$
\begin{aligned}
& \frac{\partial P_{i}}{\partial \delta_{i}}=\sum_{j \neq i}\left|V_{i}\right|\left|V_{j}\right|\left|Y_{i j}\right| \sin \left(\theta_{i j}+\delta_{j}-\delta_{i}\right) \\
& \frac{\partial P_{i}}{\partial \delta_{j}}=-\left|V_{i}\right|\left|V_{j}\right|\left|Y_{i j}\right| \sin \left(\theta_{i j}+\delta_{j}-\delta_{i}\right)
\end{aligned}
$$

where $j \neq i$.

$$
\frac{\partial Q_{i}}{\partial \delta_{j}}=\sum_{j=1}^{n}\left|V_{i} V_{j} Y_{i j}\right| \sin \left(\theta_{i j}+\delta_{j}-\delta_{i}\right)
$$

Similarly, diagonal and off diagonal elements of $J_{2}, J_{3}, J_{4}$ are:

$$
\begin{gathered}
\frac{\partial P_{i}}{\partial\left|V_{i}\right|}=2\left|V_{i}\right|\left|Y_{i i}\right| \cos \theta_{i i}+\sum_{j \neq i}\left|V_{j}\right|\left|Y_{i j}\right| \cos \left(\theta_{i j}+\delta_{j}-\delta_{i}\right) \\
\frac{\partial P_{i}}{\partial\left|V_{j}\right|}=\left|V_{i}\right|\left|Y_{i j}\right| \cos \left(\theta_{i j}+\delta_{j}-\delta_{i}\right)
\end{gathered}
$$




$$
\begin{gathered}
\frac{\partial Q_{i}}{\partial \delta_{i}}=\sum_{j \neq i}\left|V_{i}\right|\left|V_{j}\right|\left|Y_{i j}\right| \cos \left(\theta_{i j}+\delta_{j}-\delta_{i}\right) \\
\frac{\partial P_{i}}{\partial \delta_{j}}=-\left|V_{i}\right|\left|V_{j}\right|\left|Y_{i j}\right| \cos \left(\theta_{i j}+\delta_{j}-\delta_{i}\right) \\
\frac{\partial Q_{i}}{\partial\left|V_{i}\right|}=-2\left|V_{i}\right|\left|Y_{i j}\right| \sin \theta_{i i}+\sum_{j \neq i}\left|V_{i}\right|\left|Y_{i j}\right| \sin \left(\theta_{i j}+\delta_{j}-\delta_{i}\right) \\
\frac{\partial Q_{i}}{\partial\left|V_{j}\right|}=-\left|V_{i}\right|\left|Y_{i j}\right| \sin \left(\theta_{i j}+\delta_{j}-\delta_{i}\right)
\end{gathered}
$$

The terms $\Delta P_{i}$ and $\Delta Q_{i}$ in Equation (8) are the differences between the scheduled and calculated values of power called power residuals given as:

$$
\begin{aligned}
& \Delta P_{i}=P_{i}^{s c h}-P_{i} \\
& \Delta Q_{i}=Q_{i}^{s c h}-Q_{i}
\end{aligned}
$$

Using Equation (8), Equation (18) and Equation (19), $\Delta \delta_{i}$ and $\Delta\left|V_{i}\right|$ are calculated to complete the particular iteration. The new values calculated as shown below are used for the next iteration $(k+1)$

$$
\begin{gathered}
\delta_{i}^{k+1}=\delta_{i}^{k}-\Delta \delta_{i}^{k} \\
\left|V_{i}^{k+1}\right|=\left|V_{i}^{k}\right|-\left|\Delta V_{i}^{k}\right|
\end{gathered}
$$

Flow chart of Newton Raphson method is given in Figure 2. Test system i.e., IEEE 14 bus system is analyzed in Figure 3.

\section{Test System-IEEE 14 BUS System}

IEEE 14 bus system is used to validate the performance of Newton Raphson method. Table 1 and Table 2 provide the details of buses data and branches data for power system. In Table 1 , ${ }^{*}$ Bus Type (1) represents swing bus, (2) represents generator bus (PV bus) and (3) represents load bus (PQ bus).

\section{Simulation Analysis}

ETAP software is used for simulation analysis and simulation circuit is given in Figure 4. Power flow analysis for IEEE-14 bus system is carried out by Newton Raphson method. Table 3 provides the results of power flow analysis on branches of electric power system.

Table 4 gives the detail of load and losses in electric power system after power flow analysis via Newton Raphson method. IEEE-14 bus system after power flow analysis is given in Figure 5.

Power flow analysis results in generation of alerts at generators. The alerts contain the conditions of operation of generator as shown in Table 5. Conditions of operation of generators are overload, overexcited, under excited and normal. After taking into consideration the capability curve and load values, the generation values are readjusted to bring operation of generators under normal conditions and it is shown in Table 6. 


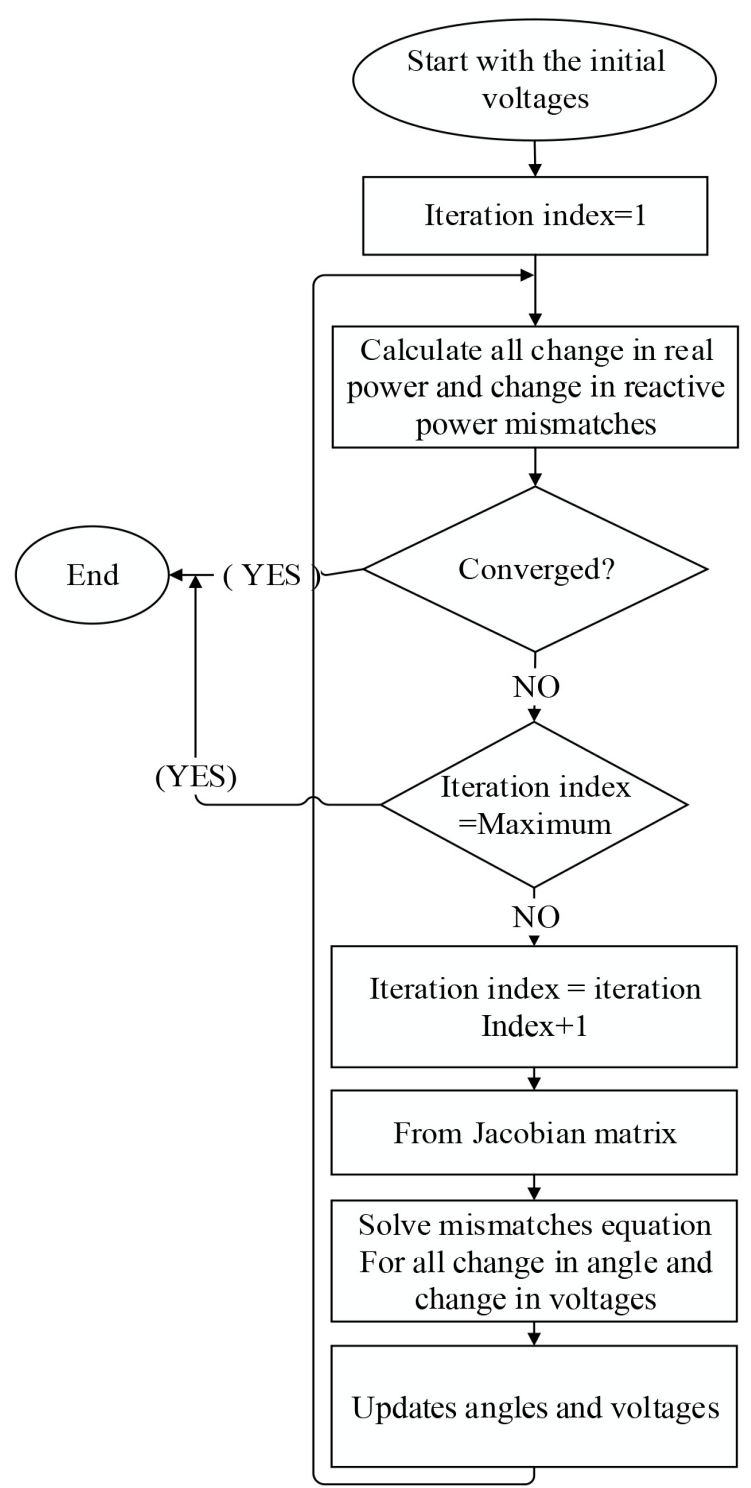

Figure 2. Flow chart of Newton Raphson method.

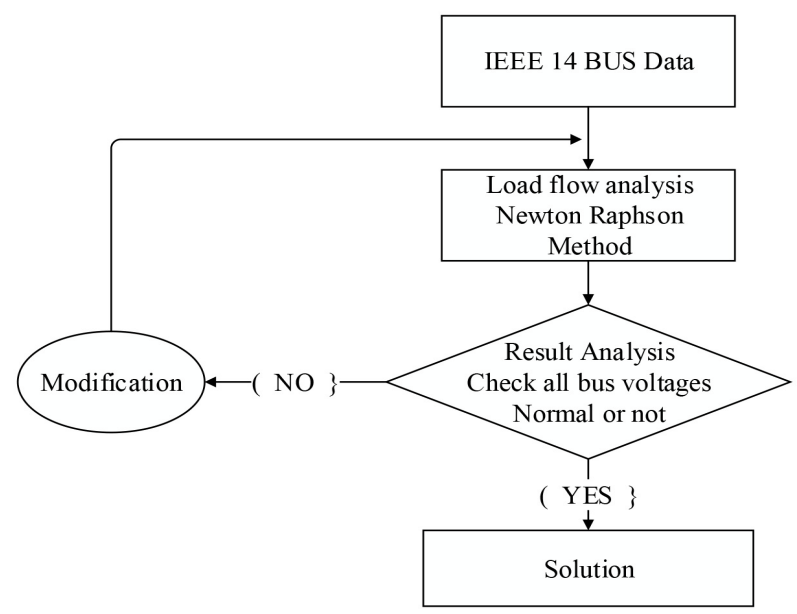

Figure 3. IEEE-14 bus system and Newton Raphson method. 
Table 1. This table shows the bus data of IEEE-14 bus system.

\begin{tabular}{|c|c|c|c|c|c|c|c|}
\hline $\begin{array}{l}\text { Bus } \\
\text { No. }\end{array}$ & $\begin{array}{c}\mathrm{P} \\
\text { GENERATED } \\
\text { (P.U) }\end{array}$ & $\begin{array}{c}\mathrm{Q} \\
\text { GENERATED } \\
\text { (P.U) }\end{array}$ & $\begin{array}{c}\mathrm{P} \\
\text { LOAD } \\
(\mathrm{P} . \mathrm{U})\end{array}$ & $\begin{array}{c}\mathrm{Q} \\
\text { LOAD } \\
(\mathrm{P} . \mathrm{U})\end{array}$ & $\begin{array}{c}\text { BUS } \\
\text { TYPE }^{*}\end{array}$ & $\begin{array}{c}\text { Q } \\
\text { GENERATED } \\
\text { MAX. (P.U) }\end{array}$ & $\begin{array}{c}\text { Q } \\
\text { GENERATED } \\
\text { MIN. (P.U) }\end{array}$ \\
\hline 1 & 2.32 & 0.00 & 0.00 & 0.00 & 2 & 10.0 & -10.0 \\
\hline 2 & 0.4 & -0.424 & 0.2170 & 0.1270 & 1 & 0.5 & -0.4 \\
\hline 3 & 0.00 & 0.00 & 0.9420 & 0.1900 & 2 & 0.4 & 0.00 \\
\hline 4 & 0.00 & 0.00 & 0.4780 & 0.00 & 3 & 0.00 & 0.00 \\
\hline 5 & 0.00 & 0.00 & 0.0760 & 0.0160 & 3 & 0.00 & 0.00 \\
\hline 6 & 0.00 & 0.00 & 0.1120 & 0.0750 & 2 & 0.24 & -0.06 \\
\hline 7 & 0.00 & 0.00 & 0.00 & 0.00 & 3 & 0.00 & 0.00 \\
\hline 8 & 0.00 & 0.00 & 0.00 & 0.00 & 2 & 0.24 & -0.06 \\
\hline 9 & 0.00 & 0.00 & 0.2950 & 0.1660 & 3 & 0.00 & 0.00 \\
\hline 10 & 0.00 & 0.00 & 0.0900 & 0.0580 & 3 & 0.00 & 0.00 \\
\hline 11 & 0.00 & 0.00 & 0.0350 & 0.0180 & 3 & 0.00 & 0.00 \\
\hline 12 & 0.00 & 0.00 & 0.0610 & 0.0160 & 3 & 0.00 & 0.00 \\
\hline 13 & 0.00 & 0.00 & 0.1350 & 0.0580 & 3 & 0.00 & 0.00 \\
\hline 14 & 0.00 & 0.00 & 0.1490 & 0.0500 & 3 & 0.00 & 0.00 \\
\hline
\end{tabular}

Table 2. This table shows the branch data of IEEE-14 bus system.

\begin{tabular}{|c|c|c|c|c|}
\hline From Bus & To Bus & Resistance (p.u.) & Reactance (p.u) & Line charging (p.u.) \\
\hline 1 & 2 & 0.01938 & 0.05917 & 0.0528 \\
\hline 1 & 5 & 0.05403 & 0.22304 & 0.0492 \\
\hline 2 & 3 & 0.04699 & 0.19797 & 0.0438 \\
\hline 2 & 4 & 0.05811 & 0.17632 & 0.0374 \\
\hline 2 & 5 & 0.05695 & 0.17388 & 0.034 \\
\hline 3 & 4 & 0.06701 & 0.17103 & 0.0346 \\
\hline 4 & 5 & 0.01335 & 0.04211 & 0.0128 \\
\hline 4 & 7 & 0.00 & 0.20912 & 0.00 \\
\hline 4 & 9 & 0.00 & 0.55618 & 0.00 \\
\hline 5 & 6 & 0.00 & 0.25202 & 0.00 \\
\hline 6 & 11 & 0.09498 & 0.1989 & 0.00 \\
\hline 6 & 12 & 0.12291 & 0.25581 & 0.00 \\
\hline 6 & 13 & 0.06615 & 0.13027 & 0.00 \\
\hline 7 & 8 & 0.00 & 0.17615 & 0.00 \\
\hline 7 & 9 & 0.00 & 0.11001 & 0.00 \\
\hline 9 & 10 & 0.03181 & 0.08450 & 0.00 \\
\hline 9 & 14 & 0.12711 & 0.27038 & 0.00 \\
\hline 10 & 11 & 0.08205 & 0.19207 & 0.00 \\
\hline 12 & 13 & 0.22092 & 0.19988 & 0.00 \\
\hline 13 & 14 & 0.17093 & 0.34802 & 0.00 \\
\hline
\end{tabular}


Table 3. This table provides the result of power flow analysis of on branches of IEEE-14 bus system.

\begin{tabular}{ccccccc}
\hline ID & Type & From Bus & To Bus & $\mathrm{R}$ & $\mathrm{X}$ & $\mathrm{Z}$ \\
\hline $\mathrm{T} 1$ & 2W XFMR & Bus5 & Bus6 & 554.70 & 832.05 & 1000 \\
T2 & 2W XFMR & Bus9 & Bus6 & 554.70 & 832.05 & 1000. \\
T5 & 2W XFMR & Bus9 & Bus4 & 69.43 & 3124.23 & 3125 \\
Z1 & Impedance & Bus1 & Bus2 & 0.00 & 0.01 & 0.01 \\
Z2 & Impedance & Bus1 & Bus5 & 0.01 & 0.04 & 0.04 \\
Z3 & Impedance & Bus3 & Bus4 & 0.01 & 0.03 & 0.03 \\
Z4 & Impedance & Bus2 & Bus3 & 0.01 & 0.04 & 0.04 \\
Z5 & Impedance & Bus2 & Bus4 & 0.01 & 0.03 & 0.04 \\
Z6 & Impedance & Bus5 & Bus4 & 0.00 & 0.01 & 0.01 \\
Z7 & Impedance & Bus9 & Bus15 & & 0.02 & 0.02 \\
Z8 & Impedance & Bus4 & Bus15 & & 0.04 & 0.04 \\
Z9 & Impedance & Bus8 & Bus15 & & 0.03 & 0.03 \\
Z10 & Impedance & Bus10 & Bus9 & 0.01 & 0.02 & 0.02 \\
Z11 & Impedance & Bus11 & Bus10 & 0.02 & 0.04 & 0.04 \\
Z12 & Impedance & Bus11 & Bus6 & 0.02 & 0.04 & 0.04 \\
Z13 & Impedance & Bus12 & Bus6 & 0.02 & 0.05 & 0.05 \\
Z14 & Impedance & Bus13 & Bus12 & 0.04 & 0.04 & 0.06 \\
Z15 & Impedance & Bus13 & Bus14 & 0.03 & 0.07 & 0.07 \\
Z16 & Impedance & Bus14 & Bus9 & 0.02 & 0.05 & 0.06 \\
Z17 & Impedance & Bus5 & Bus2 & 0.01 & 0.03 & 0.03 \\
Z18 & Impedance & Bus4 & Bus9 & & 0.11 & 0.11 \\
Z19 & Impedance & Bus13 & Bus6 & 0.13 & 0.02 & 0.13 \\
\hline & & & & & & \\
\hline
\end{tabular}

Table 4. This table shows the result of load and losses after load flow analysis at $230 \mathrm{kv}$ and at 100 percent magnitude.

\begin{tabular}{ccccc}
\hline \multirow{2}{*}{ ID } & \multicolumn{2}{c}{ Load } & \multicolumn{2}{c}{ Losses } \\
\cline { 2 - 5 } & MW & MVAR & MW & MVAR \\
\hline Bus1 & - & - & - & - \\
Bus2 & 27.880 & 17.278 & 6.970 & 4.320 \\
Bus3 & 336.600 & 208.606 & 84.150 & 52.151 \\
Bus4 & 169.320 & 104.935 & 42.330 & 26.234 \\
Bus5 & 26.520 & 16.436 & 6.630 & 4.109 \\
Bus6 & 42.840 & 26.550 & 10.710 & 6.637 \\
Bus8 & - & - & - & - \\
Bus9 & 110.840 & 68.692 & 27.710 & 17.173 \\
Bus10 & 34.000 & 21.071 & 8.500 & 5.268 \\
Bus11 & 104.720 & 64.900 & 26.180 & 16.225 \\
Bus12 & 135.320 & 83.864 & 33.830 & 20.966 \\
Bus13 & 48.960 & 30.343 & 12.240 & 7.586 \\
Bus14 & 53.720 & 33.293 & 13.430 & 8.323 \\
Bus15 & - & - & - & - \\
Total Number of & 1090.720 & 675.968 & 272.680 & 168.992 \\
Buses: 14 & & & & \\
\hline
\end{tabular}


Table 5. This table gives the condition of three phase generators after power flow analysis via Newton Raphson method.

\begin{tabular}{ccccc}
\hline Device ID & Condition & Rating/limit & Operating & \%Operating \\
\hline Gen1 & Overload & $60 \mathrm{MW}$ & 60 & 100 \\
Gen1 & Over Excited & $37.5 \mathrm{Mvar}$ & 37.185 & 100 \\
Gen2 & Overload & $25 \mathrm{MW}$ & 25 & 100 \\
Gen2 & Under Excited & 0 & 0 & - \\
Gen3 & Overload & $25 \mathrm{MW}$ & 25 & 100 \\
Gen3 & Under Excited & & 0 & - \\
Gen4 & Overload & $60 \mathrm{MW}$ & 60 & 100 \\
Gen4 & Under Excited & & 0 & - \\
Swing Gen & Overload & $615 \mathrm{MW}$ & 1193.143 & 194 \\
\hline
\end{tabular}

Table 6. This table gives the values of generation under normal operating conditions of generators.

\begin{tabular}{ccccc}
\hline Device ID & Condition & Rating/limit & Operating & \%Operating \\
\hline Gen1 & Normal & $70 \mathrm{MW}$ & $60 \mathrm{MW}$ & 87 \\
Gen1 & Normal & $37 \mathrm{Mvar}$ & $37 \mathrm{Mvar}$ & 100 \\
Gen2 & Normal & $40 \mathrm{MW}$ & $30 \mathrm{MW}$ & 70 \\
Gen2 & Normal & $15 \mathrm{Mvar}$ & $15 \mathrm{Mvar}$ & 100 \\
Gen3 & Normal & $40 \mathrm{MW}$ & $30 \mathrm{MW}$ & 70 \\
Gen3 & Normal & $16 \mathrm{Mvar}$ & $16 \mathrm{Mvar}$ & 100 \\
Gen4 & Normal & $80 \mathrm{MW}$ & $70 \mathrm{MW}$ & 87.5 \\
Gen4 & Normal & $36.5 \mathrm{Mvar}$ & $36.5 \mathrm{Mvar}$ & 100 \\
Swing Gen & Normal & $1300 \mathrm{MW}$ & 1173.151 & 90 \\
\hline
\end{tabular}

ETAP report is generated and is given in Table 7 to depict the generation and load in electric power system test model.

Voltage and current of test system are measured before and after load flow analysis to depict the state of the system and are shown in Figure 6 and Figure 7. Change in the values of voltage and angles are observed. It is the requirement of power system to have good voltage regulation for ensuring acceptable power factor value.

\section{Conclusion}

In this research paper, IEEE-14 bus system is developed and analysed. Power flow analysis is conducted through Newton Raphson method. Further, operating conditions of generators are determined. Generation values are readjusted by 


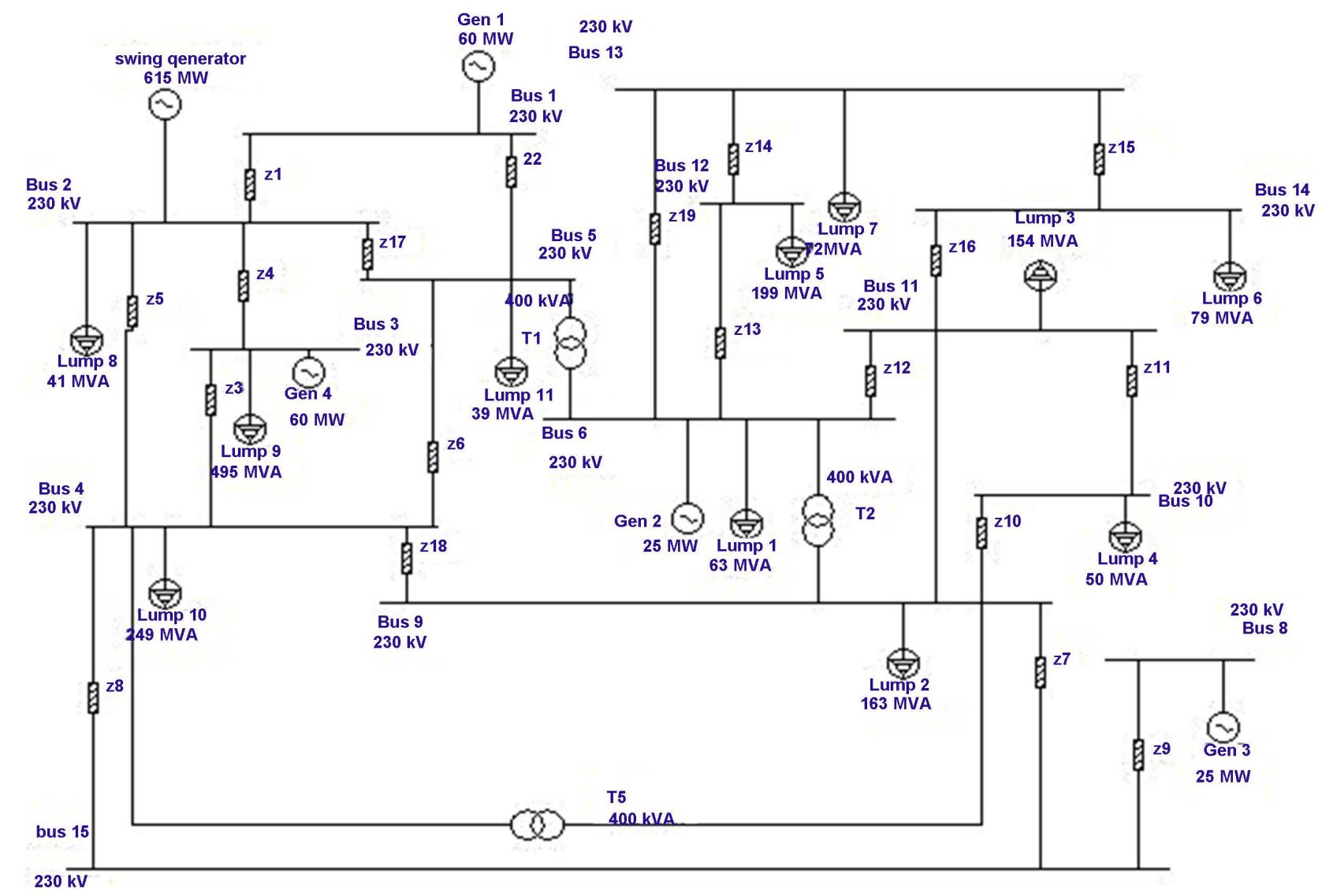

Figure 4. This figure shows the simulation circuit of IEEE-14 bus system on ETAP software.

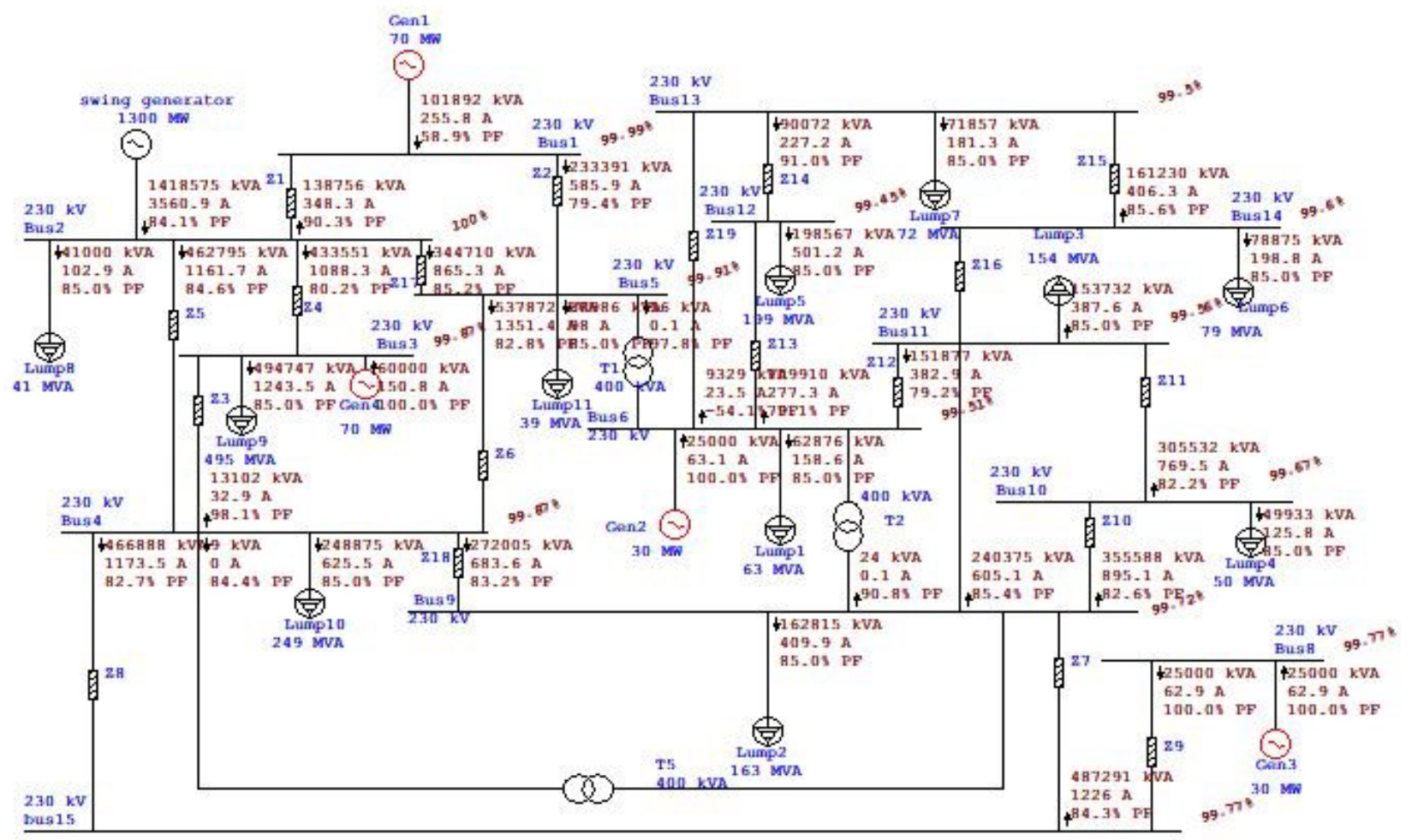

Figure 5. IEEE-14 bus system after power flow analysis. 
Table 7. This table shows the load flow ETAP software report via Newton Rahson method.

\begin{tabular}{|c|c|c|c|c|c|c|c|c|c|c|c|c|}
\hline \multicolumn{2}{|c|}{ LBUS } & \multicolumn{2}{|c|}{ VOLTAGEES } & \multicolumn{2}{|c|}{ Generation } & \multicolumn{2}{|c|}{ Load } & \multicolumn{4}{|c|}{ Load flow } & \multirow[b]{2}{*}{$\% \mathrm{PF}$} \\
\hline ID & $\mathrm{kV}$ & \% Mag. & Ang. & MW & Mvar & MW & Mvar & ID & MW & Mvar & Amp & \\
\hline \multirow[t]{2}{*}{ Bus1 } & 230.000 & 99.986 & 0.0 & 60.000 & 37.000 & 0 & 0 & Bus2 & -123.282 & --88.741 & 381.4 & 81.2 \\
\hline & & & & & & & & Bus5 & 183.282 & 125.741 & 558.0 & 82.5 \\
\hline \multirow[t]{4}{*}{ *Bus2 } & 230.000 & 100.000 & 0.0 & 1173.151 & 744.880 & 34.850 & 21.598 & Bus1 & 123.291 & 88.767 & 381.4 & 81.2 \\
\hline & & & & & & & & Bus3 & 341.401 & 233.300 & 1038.0 & 82.6 \\
\hline & & & & & & & & Bus4 & 384.927 & 230.797 & 1126.6 & 85.8 \\
\hline & & & & & & & & Bus5 & 288.683 & 170.419 & 841.5 & 86.1 \\
\hline \multirow[t]{2}{*}{ Bus3 } & 230.000 & 99.882 & -0.1 & 70.000 & 36.500 & 420.552 & 260.635 & Bus4 & -9.303 & 8.525 & 31.7 & -73.7 \\
\hline & & & & & & & & Bus2 & -341.249 & -232.660 & 1038.0 & 82.6 \\
\hline \multirow[t]{6}{*}{ Bus4 } & 230.000 & 99.881 & -0.1 & 0 & 0 & 211.549 & 131.106 & Bus3 & 9.303 & -8.525 & 31.7 & -73.7 \\
\hline & & & & & & & & Bus2 & -384.705 & -230.125 & 1126.6 & 85.8 \\
\hline & & & & & & & & Bus5 & -438.534 & -274.827 & 1300.7 & 84.7 \\
\hline & & & & & & & & Bus15 & 378.986 & 240.940 & 1128.7 & 84.4 \\
\hline & & & & & & & & Bus9 & 223.393 & 141.425 & 664.5 & 84.5 \\
\hline & & & & & & & & Bus9 & 0.008 & 0.005 & 0.0 & 85.7 \\
\hline \multirow[t]{4}{*}{ Bus5 } & 230.000 & 99.914 & 0.0 & 0 & 0 & 33.139 & 20.537 & Bus1 & -183.232 & -125.532 & 558.0 & 82.5 \\
\hline & & & & & & & & Bus4 & 438.601 & 275.040 & 1300.7 & 84.7 \\
\hline & & & & & & & & Bus2 & -288.562 & -170.055 & 841.5 & 86.2 \\
\hline & & & & & & & & Bus6 & 0.054 & 0.010 & 0.1 & 98.3 \\
\hline \multirow[t]{5}{*}{ Bus6 } & 230.000 & 99.533 & -0.3 & 30.000 & 15.000 & 53.450 & 33.126 & Bus11 & -117.751 & -82.473 & 362.6 & 81.9 \\
\hline & & & & & & & & Bus12 & 87.306 & 70.447 & 282.9 & 77.8 \\
\hline & & & & & & & & Bus13 & 7.070 & -6.081 & 23.5 & -75.8 \\
\hline & & & & & & & & Bus5 & -0.054 & -0.010 & 0.1 & 98.4 \\
\hline & & & & & & & & Bus9 & -0.022 & -0.009 & 0.1 & 92.0 \\
\hline Bus8 & 230.000 & 99.791 & -0.1 & 30.000 & 16.000 & 0 & 0 & Bus15 & 30.000 & 16.000 & 85.5 & 88.2 \\
\hline \multirow[t]{6}{*}{ Bus9 } & 230.000 & 99.732 & -0.2 & 0 & 0 & 138.402 & 85.774 & Bus15 & -408.986 & -255.651 & 1214.0 & 84.8 \\
\hline & & & & & & & & Bus10 & 291.130 & 190.366 & 875.5 & 83.7 \\
\hline & & & & & & & & Bus14 & 202.833 & 120.195 & 593.4 & 86.0 \\
\hline & & & & & & & & Bus4 & -223.393 & -140.688 & 664.5 & 84.6 \\
\hline & & & & & & & & Bus6 & 0.022 & 0.009 & 0.1 & 91.9 \\
\hline & & & & & & & & Bus4 & -0.008 & -0.005 & 0.0 & 85.8 \\
\hline \multirow[t]{2}{*}{ Bus10 } & 230.000 & 99.684 & -0.2 & 0 & 0 & 42.446 & 26.306 & Bus9 & -291.057 & -190.172 & 875.5 & 83.7 \\
\hline & & & & & & & & Bus11 & 248.610 & 163.866 & 749.8 & 83.5 \\
\hline \multirow[t]{2}{*}{ Bus11 } & 230.000 & 99.586 & -0.3 & 0 & 0 & 130.684 & 80.990 & Bus10 & -248.472 & -163.542 & 749.8 & 83.5 \\
\hline & & & & & & & & Bus6 & 117.788 & 82.551 & 362.6 & 81.9 \\
\hline \multirow[t]{2}{*}{ Bus12 } & 230.000 & 99.479 & -0.3 & 0 & 0 & 168.798 & 104.612 & Bus6 & -87.276 & -70.385 & 282.9 & 77.8 \\
\hline & & & & & & & & Bus13 & -81.522 & -34.226 & 223.1 & 92.2 \\
\hline \multirow[t]{3}{*}{ Bus13 } & 230.00 & 99.986 & -0.3 & 0 & 0 & 61.08 & 37.85 & Bus12 & 81.5 & 34.256 & 223.1 & 92.2 \\
\hline & & & & & & & & Bus14 & -135.57 & -78.194 & 394.7 & 86.5 \\
\hline & & & & & & & & Bus6 & -7.0650 & 6.081 & 23.5 & -75.8 \\
\hline \multirow[t]{3}{*}{ Bus14 } & 230.0 & 99.622 & -0.2 & 0 & 0 & 67.04 & 41.553 & Bus13 & 135.6 & 78.3 & 394.7 & 86.6 \\
\hline & & & & & & & & Bus9 & -202.69 & -119.90 & 593.4 & 86.6 \\
\hline & & & & & & & & Bus9 & 408.9 & 256.1 & 1214 & 84 \\
\hline \multirow[t]{2}{*}{ Bus15 } & 230.000 & 99.7 & -0.1 & 0 & 0 & 0 & 0 & Bus4 & -378.9 & -240.1 & 1128.7 & 84.5 \\
\hline & & & & & & & & Bus8 & -30.0 & -15.996 & 85.5 & 88.2 \\
\hline
\end{tabular}




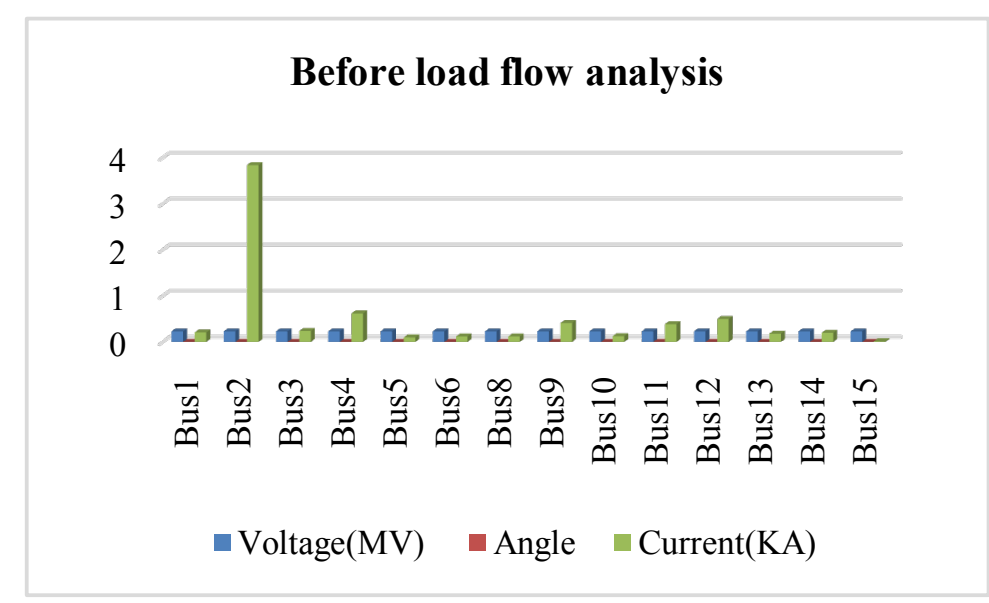

Figure 6. Graphical representation of voltage and current of IEEE-14 bus system before power flow analysis.

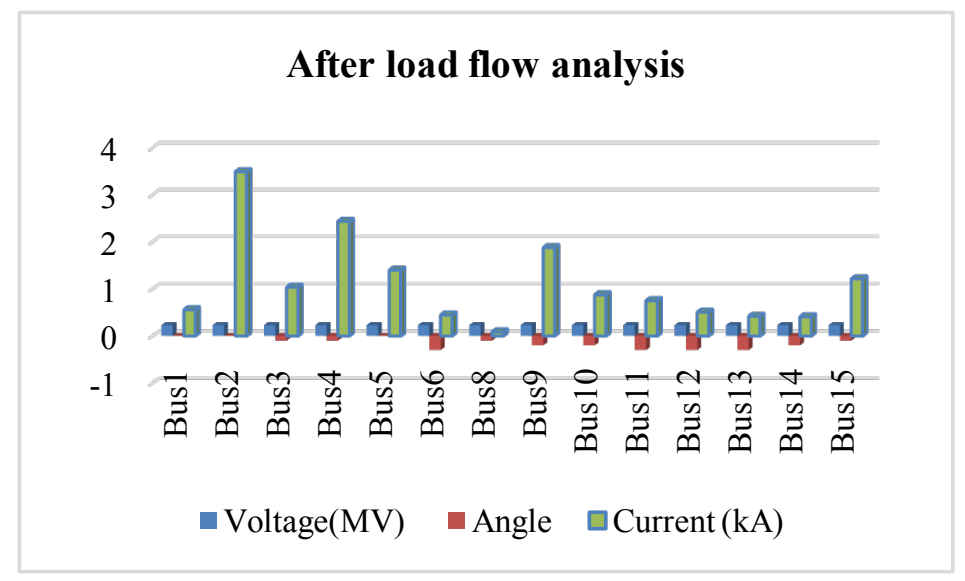

Figure 7. Graphical representation of voltage and current of IEEE-14 bus system after power flow analysis.

taking into consideration capability curve and load values to bring operation of generators under normal conditions. It is found that power flow analysis is comprehensively conducted through Newton Raphson method via ETAP software. This research depicts the importance of implementing Newton Raphson and using ETAP software at industrial and commercial levels. With a little change in voltage values at buses of power system, generators are brought to normal operating conditions. Power factor is not much compromised. Load is meeting in economic operating conditions. Weak branches are identified. Capacitor banks and static volt Ampere reactive compensator (SVC) would be the ultimate solution to make them strong. This load flow study would lead to the installation of flexible alternating current transmission system (FACTS) devices for reactive power compensation. This research led to the development of optimization algorithms for finding the location of FACTS devices. Transient and economic dispatch for circuit breaker placements and economics of generations will be the future work of this power flow analysis. 


\section{Conflicts of Interest}

The authors declare no conflicts of interest regarding the publication of this paper.

\section{References}

[1] Muzzammel, R., Ahsan, M. and Ahmad, W. (2015) Non-Linear Analytic Approaches of Power Flow Analysis and Voltage Profile Improvement. 2015 Power Generation System and Renewable Energy Technologies (PGSRET), Islamabad, June 2015, 1-7.

[2] Saadat, H. (1999) Power System Analysis. Mc Graw-hill, Inc., 2 Pennsylvania Plaza New York City.

[3] Stevenson, W.D. (1982) Elements of Paver System Analysis. McGraw-Hill, 2 Pennsylvania Plaza New York City.

[4] Athay, T. (1983) An Overview of Power Flow Analysis. 1983 American Control Conference, San Francisco, CA, USA, 22-24 June 1983, 404-410. https://doi.org/10.23919/ACC.1983.4788145

[5] Mešanović, A., Münz, U. and Ebenbauer, C. (2018) Robust Optimal Power Flow for Mixed AC/DC Transmission Systems with Volatile Renewables. IEEE Transactions on Power Systems, 33, 5171-5182. https://doi.org/10.1109/TPWRS.2018.2804358

[6] Levron, Y., Guerrero, J.M. and Beck, Y. (2013) Optimal Power Flow in Microgrids with Energy Storage. IEEE Transactions on Power Systems, 28, 3226-3234. https://doi.org/10.1109/TPWRS.2013.2245925

[7] Stott, B. (1974) Review of Load-Flow Calculation Methods. Proceedings of the IEEE, 62, 916-929. https://doi.org/10.1109/PROC.1974.9544

[8] Fikri, M., Cheddadi, B., Sabri, O., Haidi, T., Abdelaziz, B. and Majdoub, M. (2018) Power Flow Analysis by Numerical Techniques and Artificial Neural Networks. 2018 Renewable Energies, Power Systems \& Green Inclusive Economy (REPS-GIE), Casablanca, 23-24 April 2018, 1-5. https://doi.org/10.1109/REPSGIE.2018.8488870

[9] Montoya, O.D., Garrido, V.M., Gil-González, W. and Grisales-Noreña, L. (2019) Power Flow Analysis in DC Grids: Two Alternative Numerical Methods. IEEE Transactions on Circuits and Systems II: Express Briefs, 1-1.

https://doi.org/10.1109/TCSII.2019.2891640

[10] Asrari, A. and Ramos, B. (2018) Power System Protection Upgrade at Anclote Plant a Case Study in Florida State. 2018 IEEE Texas Power and Energy Conference (TPEC), College Station, TX, 8-9 February 2018, 1-6. https://doi.org/10.1109/TPEC.2018.8312091

[11] Chandraratne, C., Woo, W.L., Logenthiran, T. and Naayagi, R.T. (2018) Adaptive Overcurrent Protection for Power Systems with Distributed Generators. 2018 th International Conference on Power and Energy Systems (ICPES), Colombo, Sri Lanka, 21-22 December 2018, 98-103. https://doi.org/10.1109/ICPESYS.2018.8626908

[12] Soni, C.J., Gandhi, P.R. and Takalkar, S.M. (2015) Design and Analysis of $11 \mathrm{KV}$ Distribution System Using ETAP Software. 2015 International Conference on Computation of Power, Energy, Information and Communication (ICCPEIC), Chennai, Chennai, 22-23 April 2015, 0451-0456. https://doi.org/10.1109/ICCPEIC.2015.7259526 
[13] Alinejad-Beromi, Y., Sedighizadeh, M., Bayat, M.R. and Khodayar, M.E. (2007) Using Genetic Alghoritm for Distributed Generation Allocation to Reduce Losses and Improve Voltage Profile. 2007 42nd International Universities Power Engineering Conference, Brighton, 4-6 September 2007, 954-959.

https://doi.org/10.1109/UPEC.2007.4469077 